\title{
Status and development trends of Pu'er tea industrial mechanization and automation
}

\author{
Linlin Yang, Xiuying Tang, Jie Shi, Wangyun Ning and Qiong Wang \\ Faculty of Engineering and Technology \\ Yunnan Agricultural University, Kunming \\ Yunnan ,China \\ Email: kgy200398@126.com
}

\begin{abstract}
The general trend of tea technology development is tea industrial whole process automation. Pu'er tea with unique processing technology, its equipment with other tea varieties are very different. This paper summarizes the Pu'er tea industry mechanization and automation status, analysis of the tea industry automation process problems, proposed solutions and pointed out the development trend of tea industry automation.
\end{abstract}

Keywords- Tea garden; management; fermentation tank

\section{INTRODUCTION}

Pu'er tea is a special tea, it is a loose or compressed tea through the fermentation process with the raw materials of large-leafed Maocha. Pu'er tea appearance color red brown, thick bright endoplasmic liquor color, aroma and unique flavor, taste mellow back to gan. " The older the better " is recognized as the Pu'er tea is differentiated from other tea features the largest incense. At present the domestic tea processing largely remain in the traditional processing mode, manual mill has larger proportion. A certain scale of production enterprises also are existing processing equipment is pallet, production technology workers basic adopy experience processing, and influenced by season and environment conditions, which makes the tea quality stability difference, it is difficult to meet the market for highquality product demand. But in recent years with the development of automation technology continues to progress and Pu'er tea process improvement, especially the application of computer technology, making tea industry mechanization, automation of a substantial progress.

\section{Tea Garden Management Automation}

Tea garden management is the premise that raises economic benefits in tea production. Tea garden management mainly includes the tea plantation, tea cultivation, tea tree pruning, irrigation, tea-leaf picking and transport operations. At present, the tea garden management to do the best in Japan and Taiwan. The Japanese tea garden by most of farmer of dispersive management, but the tea concentration piece, dimensions changes rate very high. For example, from the tea cultivation, standardized cultivation, convenient operation of machinery. Tea cultivation, pruning, and picking realizes mechanized operation.Japan is now more common use of tea garden management machine driven by hydraulic pressure, walking caterpillar device, cultivation, fertilization, pruning, picking in different tea garden management work need only change the work parts. Pu'er tea garden management automation level is very low, and Japan, compared, apparent lag. Most tea plantation, the artificial management way, not realized automation, using only a few simple, easy to operate, affordable manual operation machine.

\section{MMEchanization And Automation Of PU’ER TEA PRODUCTION}

Compared to other varieties of tea industry, tea machinery at home and abroad to the development of a late start, the automation, standardization, industrialization rate is low. Pu'er tea processing technology is divided into the traditional tea production techniques and cooked tea production technology. Tea production process : fixing rolling - dry - autoclaved - drying. Cooked tea production process to make - rolling - fermentation -- autoclaved drying. Fresh picked tea, by fixing, kneading, dried after, be Er Mao qing. Er Mao Qing tea production technology and green tea are basically the same, the main use of mechanical part for rolling process.In the individual farmers and small processing factory is mainly manual kneading.But in the subsequent 40 years, tea production technology is no big improvement, until now production enterprises has been in accordance with the traditional method of producing Pu'er tea, because tea production technology on the staff of production experience and professional level has a very high demand, and Pu'er tea fermentation process exist in health conditions difference, unstable quality, productivity is low problem, all these have a serious impact on the tea industry health, continuously, stable development. However, with the development of automation technology, as well as several patent techniques and the advent and tea production line makes the Pu'er tea production automation is possible.

\section{A. Double moisturizing rotation type Pu'er tea fermentation tank}

Hongjie Zhou, Yunzhan Huang, Xiaoqiang Wu invented a double moisturizing rotation type Pu'er tea fermentation tank ( Patent No. ZL2009201116889 ), the various parts of the structure as shown in figure 1. Patent double stainless steel tank insulation structure, both inside and outside the body is a sandwich, outer tank closure, inner tank is provided with a small hole, seepage and ventilatory function, and guarantee the fermentation environment is not subject to 
external environmental conditions, can achieve the Pu'er tea fermentation of moisturizing effect. The support shaft and a stirring rod is a hollow, holes, 4 stirring rods are uniformly distributed on the supporting shaft, convenient fermentation when ventilation and humidity regulator. Fermentation tank work, motor drives the inner layer and the outer layer of the roller to rotate, a stirring rod stirring up the turning effect.Fermentation tank automatic control system using PLC as the controller, and is connected with the transducer. Fermentation tank automatic control system by frequency conversion device, precise and quantitative device, the temperature and humidity sensor and $\mathrm{pH}$ sensor. By using the patent device, can simultaneously realize tea fermentation process in precise quantitative tide, ventilation, temperature control, humidity control, data acquisition, data storage, data analysis and different fermentation stage of deblocking and replenishment functions control. The patent has the following characteristics: (1) Experiments show that, using the device can shorten the production cycle and half, reduce loss, improve tea rate, reduce costs; ( 2) The device for ground type, abandons the traditional fermentation process of ground, clean, improve the Pu'er tea fermentation health status; ( 3 ) Factors of fermentation and quality changes between scientifically controlled, making short fermentation period, and process control. Mixing structure instead of turning behavior for Puer tea uniform tide provided a guarantee, automatic control device to replace manual control the fermentation process automation.

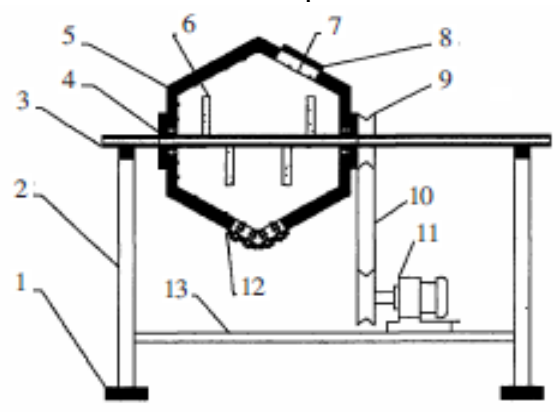

1Base; 2Bracket; 3Support shaft; 4Bearing parts; 5The outer cylinder; 6 Stirring rod with hole ; 7Inner cylinder; 8Feed inlet; 9Pulley; 10Leather belt; 11Motor; 12Drainage outlet ; 13The fixed rod

Figure 1. Double moisturizing rotation type Pu'er tea fermentation tank structure diagram

\section{B. Pu'er tea fermentation clean workshop}

Pu'er tea fermentation workshop using modern measurement and control technology and equipment, by the computer processing system ( man-machine interface display ), PLC master device, transmitter, sensors ( includes a humidity sensor, humidity sensor ), automatic alarm system, intelligent humidification equipment etc. Its working principle is: temperature, humidity sensor for real-time detection, acquisition of Pu'er tea fermentation workshop environment temperature, humidity data. When the environment temperature, humidity is higher than the set temperature, humidity value atomization humidification equipment and heating equipment automatic stop and start the fan [16]; when the environmental humidity is lower than the minimum humidity value atomization humidification equipment automatically open, reaching the highest humidity value is closed; when the environment temperature is lower than the minimum temperature heating device for automatic opening, reaches the maximum temperature value close. Clean production workshop has the following characteristics: (1) Sampling of data real-time and automatically. Sensor instead of the thermometer, hygrometer and tea water oven method analysis, equipped with monitoring software, process parameter display timely and accurately, variable artificial measurement automatic measurement, labor saving; ( 2) the workshop environment artificial controllable. Not affected by seasons, so that different batches of Pu'er tea quality tends to be stable; ( 3 ) control of water temperature control automation. (4) turning more accurate. Alarm can remind the staff, avoid tea pile shovel do not lead to timely and worry, carbonization. Construction of Puer tea fermentation clean workshop has important significance, laid the Pu'er tea clean production, automation and standardization of the foundation, provides the Pu'er tea quality inspection theory, for the tea quality promotion created good condition.

\section{Pu'er tea fermentation device automation}

China's first automatic Puer tea fermentation equipment production line in January 16, 2012 in the city of Pu'er put into use, the project won the three national invention patents, two utility model patents. Compared with traditional artificial fermentation, the use of automated production line equipment can be shortened by half fermentation fermentation time, and the product quality is very stable, ensure the Pu'er tea quality, health and safety. Realization of the Pu'er tea standardized production, the annual production capacity reached 1000 tons.

\section{TEA INDUSTRY AUTOMATION TRENDS}

\section{A. Tea Machinery Standardization}

Compared to other varieties of tea machinery, tea industry machinery research started late, Pu'er tea processing professional equipment lack of a unified technical standards. In addition to standard parts, GM parts, the production of tea machine parts can not be universal, on one hand brought huge inconvenience to the user, but also increase the burden and cost of manufacturing enterprises, to some extent hindered the tea machinery continuous, automatic development. Therefore, tea machinery must have the development, must develop a unified standard, realize the general.

\section{B. From simple mechanical to automation and intelligent}

After years of development, Pu'er tea-producing areas in some places has been the realization of a simple mechanical, some models have also achieved a simple automation, but only limited to stand-alone operations, from the mechanical and electrical integration big gap between. Although in recent years a variety of tea garden management machine and tea processing machinery has been developed, but it is difficult to find a high technology content, can realize 
automatic processing, continuous production of tea industry machinery, on the tea industry machinery and nothing more than that. With the Pu'er tea tea basic theory research is unceasingly thorough, tea new technology, new technology, new equipment will continue to come out, intelligence, automation will Pu'er tea is the development trend of equipment.

\section{Reform of Puer tea processing technology}

Pu'er tea process largely determines the Pu'er tea processing equipment, Pu'er tea equipment improvement also affected the Pu'er tea processing technology. Therefore, the reform of Pu'er tea processing technology to a certain extent, the promotion of tea equipment development. Combined with the food standardization requirements, making the implementation of clean processing technology procedures, the Pu'er tea production workshop design, construction of new tea plant and introduction of new tea processing equipment to make tea processing production specifications and standards is the trend of the development of Pu'er tea, and tea industry development trend of mechanization automation.

\section{CONCLUSION}

Pu'er tea Yunnan food industry as a special industry, it is an economy, the development of tea industry to a certain extent, promote the economic development of Yunnan province. But our country tea processing mechanization, automation line and Western Europe, Japan and other developed countries, there is still a great gap between the Pu'er tea industry, low level of mechanization and automation has seriously restricted the further development of tea industry. Therefore, to strengthen the Pu'er tea cultivation, planting, fertilizing, picking, processing production machinery of the development, realization of Pu'er tea industry wide mechanization, automation, is enhances the Pu'er tea market competitiveness, promote the tea industry health, orderly development is an important condition, thus can make the tea industry from extensive model to intensive model change. In addition, the development of tea industry will contribute to promoting agricultural synergism, farmer is added close, especially in China's current rural labor shortage of sex of structure of resource condition, development of tea production mechanization, automation, not only the sense is great, but also very urgent.

\section{REFERENCES}

[1] Hongbo Liu,Yunzhan Huang. “Study on Current Development and Development Strategy of the Modernization of Pu-erh Tea Processing Equipment” Journal of Anhui Agri. Sci. 2012, 40( 15) : $8773-$ 8775.

[2] Yunzhan Huang, Hongjie Zhou, Yongjie Zhao,etc. “The research and developmenton Pu'er tea fermentation automatic control technology and key equipment " Proceedings of 2010 3rd IEEE International conference on Computer Science and InformationTechnology. Beijing China:Institute of Electrical and Electronics Engineers, Inc. 2010:555 - 559.

[3] Shaoshuai Wu, Yali Li, Yunzhan Huang, etc. "Research and development of the patent technology on automatic fermentation of Pu'er tea”.Food and machinery. Vol.27,No.6, Nov. 2011:183-185.

[4] Yongjie Zhao, Yunzhan Huang, Hongjie Zhou,etc. "Design and inplementation of measurement and control system for pu'er tea fermentation workshop" . Food and machinery. Vol.28,No.2, Mar. 2012:98-101

[5] Yunzhan Huang, Yongjie Zhao, Yali Li, etc. "Study on application and development of fermentation tank for pu'er tea”. Vol.28,No.1, Jan. 2012:115-174.

[6] Zhengang Ma, Yunzhan Huang, Hongjie Zhou, etc. "Lift-off type pu'er tea fermentation pot's design and automatic control research"2010 3rd international conference on Advanced Computer Theory Engineering Chengdu,china. Institute of Electrical and Electroning Engineers, Inc,2010:566-570.

[7] Zhengang Ma, Yunzhan Huang, Hongjie Zhou, etc. "Design of Clear Pu-erh Tea Fermenting Workshop and the Environment Humidity Control" Journal of Yunnan Agricultural University, 2011, 26 ( 3) : 395 - 399T. Copper Engineer, pp. 54-60, Jan 2011. 